\title{
Evaluation of the Mini Nutritional Assessment in the elderly, Tehran, Iran
}

\author{
Bahareh Amirkalali, Farshad Sharifi, Hossein Fakhrzadeh*, Mojde Mirarefin, \\ Maryam Ghaderpanahi and Bagher Larijani \\ Endocrine and Metabolism Research Center, Tehran University of Medical Sciences, Shariati Hospital, \\ North Kargar Street, Tehran 1411713137, Islamic Republic of Iran
}

Submitted 9 July 2009: Accepted 20 January 2010: First published online 1 March 2010

\begin{abstract}
Objective: To determine whether the Mini Nutritional Assessment (MNA) can screen and diagnose for malnutrition in the Iranian elderly.

Design: The MNA was administered to all volunteers. Each patient underwent anthropometric and serum albumin measurements. Reliability, validity, sensitivity, specificity, positive- and negative-predictive values were estimated. To identify optimal threshold values for predicting malnutrition, receiver-operating characteristic curve analysis was performed for MNA scores.

Setting: Kahrizak Charity Foundation (Tehran, Iran).

Subjects: Two hundred and twenty-one consecutive elderly patients entered into the cross-sectional study. Amputees and patients with liver or renal disorders, oedema or any end-stage diseases were excluded.

Results: According to MNA score, $3 \cdot 2 \%$ were malnourished, $43 \cdot 4 \%$ were at risk of malnutrition and $53.4 \%$ were well nourished. The proportions in these categories according to ideal body weight and serum albumin were $2 \cdot 3 \%, 17 \cdot 1 \%$ and $80 \cdot 6 \%$, respectively. Cronbach's $\alpha$ coefficient (reliability) was $0 \cdot 61$. The correlations between total MNA score, anthropometric values and serum albumin (criterionrelated validity) were all significant. There were significant differences in total MNA score between two BMI groups but not between two categories according to serum albumin and skin ulcers (construct validity). The sensitivity and specificity of the MNA according to its established cut-off points were $82 \%$ and $63 \%$, respectively. Positive-predictive value was $35 \%$ and negative-predictive value was $93 \%$. By using the best cut-off point (MNA score of 22 according to Youden index), the sensitivity, specificity, positive-predictive value and negative-predictive value were $88 \%, 62 \%$, $57 \%$ and $89 \%$, respectively.

Conclusions: The MNA with its established cut-off points may not be a good fit for Asian populations, including Iranian elderly.
\end{abstract}

Malnutrition is a frequent and serious problem in geriatric patients. In ill elderly subjects it is one of the most common and least-heeded problems in hospitals and nursing homes ${ }^{(1-4)}$. The prevalence of undernutrition among older patients in nursing homes and hospitals reaches high levels, 30-60\%(5). According to a recent review, a high prevalence of malnutrition is reported in hospitalized and institutionalized elderly patients: 23 (SE 0.5) \% (range 1-74\%) in hospitals (thirty-five studies, 8596 elderly subjects) and 21 (SE 0.5 ) \% (range 5-71\%) in institutions (thirty-two studies, 6821 elderly subjects). An even higher prevalence of risk of malnutrition is observed in the same populations: $46(\mathrm{se} 0 \cdot 5) \%$ (range 8-63\%) and 51 (se $0 \cdot 6$ ) \% (range $27-70 \%$ ), respectively. In cognitively impaired elderly patients (ten studies, 2051 elderly subjects), the prevalence of malnutrition was 15 ( $\operatorname{SE~} 0 \cdot 8$ ) \% (range 0-62\%) and $44(\operatorname{SE~} 1 \cdot 1) \%$ (range 19-87\%) were at risk of malnutrition ${ }^{(6)}$. Different studies have also suggested that malnutrition is an important predictor of morbidity and mortality in the elderly ${ }^{(7,8)}$.

There is no gold standard for evaluating nutritional status $^{(9)}$; it is difficult to determine undernutrition or at risk for undernutrition, because there is a lack of consensus on how to define undernutrition ${ }^{(10,11)}$. This has led to a variety of different diagnostic criteria as well as the use of different reference values ${ }^{(12)}$.

Since its publication in $1994^{(13)}$, the Mini Nutritional Assessment (MNA) has been increasingly employed worldwide for the brief evaluation of older persons' nutritional status, which has been recommended by the European Society for Clinical Nutrition and Metabolism ${ }^{(6,14,15)}$. The MNA is composed of an anthropometric 
assessment, a brief questionnaire about diet characteristics, global health and environment, and a self-evaluation of health and nutritional state. The final score classifies nutritional state as 'well nourished' (scores higher than 23.5), 'at risk for undernutrition' (scores from 17 to 23.5) and 'undernourished' (scores lower than 17). Most published studies show the MNA to have high sensitivity and specificity and good predictive value for higher mortality, hospital admissions and other adverse outcomes ${ }^{(6,16-18)}$. Populations of different countries are heterogeneous in anthropometric and nutritional characteristics, which make the evaluation of this test in one country not readily applicable to other ones. In a Chilean population, for instance, the MNA failed to identify persons at risk for undernutrition $^{(18)}$. The MNA has not been validated in the Iranian elderly, and thus whether the MNA and its established cut-off points for the diagnosis of malnutrition and at-risk status are applicable to the Iranian elderly remains unknown. In the present study we examined whether the MNA can screen and diagnose for malnutrition and risk for malnutrition in the elderly population in the Kahrizak Charity Foundation (Tehran, Iran).

\section{Experimental methods}

\section{Participants}

The study was performed in Tehran, Iran, in 2008. Two hundred and twenty-one elderly patients entered into the present cross-sectional study consecutively by using their records in the Kahrizak Charity Foundation. The inclusion criteria were $\geq 65$ years of age, having the ability to communicate and the strength to carry through an interview, and informed consent. Amputees were excluded. Patients were excluded if they had liver or renal disorders, oedema or any end-stage diseases because these conditions affect serum albumin level. In forty-one cases serum albumin could not be measured because of insufficient blood sample and in five cases height measurement could not be performed.

\section{Nutritional assessment}

As there is no one biochemical or one anthropometric parameter to be used as a gold standard to define malnutrition, undernutrition is usually defined if two, i.e. one anthropometric measure and one biochemical analysis, or more of the variables are subnormal ${ }^{(19,20)}$. In the present study serum albumin and percentile of ideal body weight (IBW) were used to identify malnourished, at risk of malnutrition and well-nourished elderly people

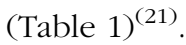

Severe and moderate protein-energy malnutrition $(\mathrm{PEM})$, protein malnutrition (PM) and energy malnutrition (EM) were classified as malnutrition; and mild PEM, EM and PM were classified as at risk of malnutrition.

IBW was calculated using the Hamwi equation ${ }^{(22)}$ as follows.

For men: $\mathrm{IBW}=48 \mathrm{~kg}+1 \mathrm{~kg}$ for every centimetre more than $150 \mathrm{~cm}$ of height

For women: IBW $=45 \mathrm{~kg}+0.9 \mathrm{~kg}$ for every centimetre more than $150 \mathrm{~cm}$ of height

$+10 \%$ for large skeleton; - $10 \%$ for small skeleton

The following equation was used to determine skeleton $\operatorname{size}^{(22)}$ :

$$
r=\text { height }(\mathrm{cm}) / \text { hand wrist circumference }(\mathrm{cm})
$$

Men have small, medium and large skeletons if $r>10 \cdot 4$, $r=9 \cdot 6-10 \cdot 4$ and $r<9 \cdot 6$, respectively. For women small, medium and large skeletons are defined as $r>11 \cdot 0$, $r=10 \cdot 1-11 \cdot 0$ and $r<10 \cdot 1$, respectively.

The full-form MNA was administered to all the volunteers. For research purposes the full test was applied to every volunteer, despite the score in the first part of the test. Each patient underwent a clinical examination including measurement of mid-arm circumference (MAC), calf circumference (CC), weight and height. Weight was recorded to the nearest $0 \cdot 1 \mathrm{~kg}$, with the subject in light clothes and barefoot, using a three-lever scale calibrated with $1 \mathrm{~kg}$ and $5 \mathrm{~kg}$ standard weights after each measurement. Height was recorded to the nearest $0 \cdot 1 \mathrm{~cm}$ using a flexible inextensible tape, with the subject's bare feet close together, back and heels against the wall, standing erect and looking straight ahead. To measure MAC the mid-point between the tip of the acromion and the olecranon process was marked while the subject held the forearm in horizontal position. The measurement was performed on the subject's arm hanging freely along the trunk with a flexible inextensible tape. CC was measured at the maximal circumference between the ankle and the knee with a flexible tape measure, manipulated to maintain close contact with the skin without compression of underlying tissues. These measures were performed on the non-dominant arm and leg. BMI was calculated

Table 1 Nutritional classification based on percentile of ideal body weight and serum albumin in elderly people ${ }^{(21)}$

\begin{tabular}{|c|c|c|c|c|}
\hline Serum albumin (g/dl) & $<60 \%$ IBW & $60-70 \%$ IBW & $71-90 \%$ IBW & $>90 \%$ IBW \\
\hline$<2.5$ & Severe PEM & Severe PEM & Moderate PEM & Severe PM \\
\hline $2 \cdot 5-3 \cdot 0$ & Severe PEM & Moderate PEM & Moderate PEM & Moderate PM \\
\hline $3 \cdot 1-3 \cdot 5$ & Moderate PEM & Moderate PEM & Mild PEM & Mild PM \\
\hline$>3 \cdot 5$ & Severe EM & Severe EM & Mild to moderate EM & Well nourished \\
\hline
\end{tabular}

IBW, ideal body weight; PM, protein malnutrition; PEM, protein-energy malnutrition; EM, energy malnutrition. 
as body weight in kilograms divided by the square of height in metres. Serum albumin was measured using the bromocresol green method with a Pars Azmoon kit (Tehran, Iran). All interviews and anthropometric measurements were performed by trained nutritionists.

\section{Statistical analyses}

Descriptive results are presented as means and standard deviations, frequencies and 95\% confidence intervals. The Kolmogorov-Smirnov test was used to check the normal distribution of variables.

As a measure of the reliability of the MNA, homogeneity was computed using Cronbach's $\alpha$ coefficient and Spearman's rank correlation coefficients between each MNA item and the total score. The correlation of the individual item was calculated when that particular item had been omitted from the total instrument ${ }^{(23,24)}$. The item-total correlation should be between $0 \cdot 20$ and $0 \cdot 80^{(23)}$, and Cronbach's $\alpha$ should be between $0 \cdot 70$ and $0 \cdot 90$. For group-level comparisons a value of $0 \cdot 70$ is usually adequate ${ }^{(23,24)}$.

Criterion-related validity is obtained when the instrument correlates highly with another criterion in the same area $^{(23)}$. To assess criterion-related validity, Spearman's rank correlation coefficients between total MNA score obtained and the criteria of BMI, serum albumin level, MAC and CC were calculated.

Concurrent validity is a type of criterion-related validity and can be used when the instrument and another measure are compared, i.e. correlated, at the same time ${ }^{(23)}$. Spearman's rank correlation coefficients were used for assessing this validity by comparing the patient's own assessment of his/her nutritional status and total MNA score obtained.

For assessing construct validity, total MNA scores were compared between patients with $\mathrm{BMI}<24$ and $\geq 24 \mathrm{~kg}$ / $\mathrm{m}^{2}$, patients with pressure sores or other skin ulcers and those without, and patients with serum albumin $<3.5$ and $\geq 3.5 \mathrm{~g} / \mathrm{dl}$.

A BMI of $24-29 \mathrm{~kg} / \mathrm{m}^{2}$ is a recommended reference interval for individuals over 65 years ${ }^{(25)}$ and a serum albumin level of $3.5 \mathrm{~g} / \mathrm{dl}$ as the cut-off point to define malnutrition has been widely accepted in previous studies ${ }^{(26,27)}$.

Differences between the groups were tested using the Mann-Whitney $U$ test (two-tailed significance).

Sensitivity (the ability to identify cases correctly, i.e. the true positives), specificity (the ability to identify non-cases correctly, i.e. the true negatives), positive-predictive value
$(\mathrm{A} /(\mathrm{A}+\mathrm{B}))$ and negative-predictive value $(\mathrm{D} /(\mathrm{C}+\mathrm{D}))$ were estimated $^{(23)}$ (see Table 2). Malnutrition and at risk of malnutrition were merged into one group and compared with the well-nourished group.

Positive-predictive value here is the probability that a patient is malnourished or at risk of malnutrition, e.g. with a positive screening result. Negative-predictive value is the probability that a patient is not malnourished or at risk of malnutrition, e.g. with a negative screening result ${ }^{(28)}$.

To identify optimal threshold values for predicting malnutrition, receiver-operating characteristic (ROC) curve analysis was performed for MNA scores. The area under the ROC curve (AUC) was also evaluated. An AUC value of 0.5 indicates that the variable performs no better than chance, whereas a value of 1.0 indicates perfect discrimination. A larger AUC represents a greater reliability and discrimination of the scoring system ${ }^{(29)}$. Cut-off values can be set depending on the purpose for which the scales are used. For screening purposes, a high sensitivity and a high negative-predictive value are required, whereas diagnosis requires a high specificity and a high-positive predictive value ${ }^{(30)}$. The best Youden index (sensitivity + specificity -1$)$ was used to determine the best cut-off point ${ }^{(30)}$. The Youden index is used to compare the proportion of cases correctly classified. The higher the Youden index, the more accurate the prediction (higher true positives and true negatives, and fewer false positives and false negatives) at the cut-off point.

$P$ values lower than 0.05 were considered significant. All computations were performed using the SPSS statistical software package version 11 (SPSS Inc., Chicago, IL, USA). Ethical approval for the study was granted by Tehran University of Medical Sciences.

\section{Results}

Eighty-nine (40.3\%) men and $132(59 \cdot 7 \%)$ women participated in the present study. Their ages ranged between 65 and 102 years; mean age was $78 \cdot 1(\mathrm{SD} 7 \cdot 5$ ) years. The most frequent medical diagnoses among the participants were CVD (42.1\%) and hypertension (57.9\%). Total MNA scores averaged $23 \cdot 6(\mathrm{sD} 3 \cdot 1)$ and ranged from a minimum of 11.5 to a maximum of $29 \cdot 5$. According to the original cut-off point of the full MNA, $3 \cdot 2 \%$ had an MNA score lower than 17 (malnourished), $43 \cdot 4 \%$ had an MNA score between 17 and 23.5 (at risk of malnutrition) and $53.4 \%$ had a score of at least 24 (well nourished). These results according to

Table 2 Cross table for calculating sensitivity, specificity and predictive values

\begin{tabular}{lcc}
\hline & $\begin{array}{c}\text { Malnourished or at risk } \\
\text { (based on \%IBW and serum albumin) }\end{array}$ & $\begin{array}{c}\text { Well nourished } \\
\text { (based on \%lBW and serum albumin) }\end{array}$ \\
\hline Malnourished or at risk (based on MNA) & A & B \\
Well nourished (based on MNA) & C & D \\
\hline
\end{tabular}

\%IBW, percentile of ideal body weight; MNA, Mini Nutritional Assessment. 
percentile of IBW and serum albumin were $2 \cdot 3 \%, 17 \cdot 1 \%$ and $80 \cdot 6 \%$, respectively. Thus classification of the nutritional status of patients according to the MNA was significantly different from classification according to serum albumin and IBW percentile $(P<0 \cdot 001)$. Using the MNA classified more people as malnourished or at risk of malnutrition.

\section{Reliability}

The reliability of the MNA is reflected in the item-total score correlations displayed in Table 3. Cronbach's $\alpha$ was $0 \cdot 61$. Eight out of eighteen items (numbers 4, 7, 8, 9, 10, 11, 12 and 14) were not in relation to the total scale. Excluding these items from the MNA increased Cronbach's $\alpha$ by $0 \cdot 02$ only.

\section{Validity}

Criterion-related validity of the MNA, assessed as the correlations between total MNA score and BMI, MAC, CC and serum albumin, is shown in Table 4. All correlations were significant.

Concurrent validity of the instrument is shown in the correlation between total MNA scores and the patients' views of their own nutritional status, which reached a statistically significant value of $r_{\mathrm{S}}=0.30 \quad(P<0.001$; Table 3).

Construct validity of the MNA was calculated, comparing total MNA scores between patients with BMI $<24$ and $\geq 24 \mathrm{~kg} / \mathrm{m}^{2}$, between patients with pressure sores or other skin ulcers and those without, and patients with serum albumin $<3.5$ and $\geq 3.5 \mathrm{~g} / \mathrm{dl}$ (Table 5 ). There were significant differences between the two BMI groups $(P<0 \cdot 001)$, but not between groups categorized according to serum albumin and skin ulcers.

The ROC curves shown in Figs 1 and 2 plot the sensitivity $v .1-$ specificity for total MNA score in predicting low serum albumin $(<3.5 \mathrm{~g} / \mathrm{dl})$ and low BMI $\left(<24 \mathrm{~kg} / \mathrm{m}^{2}\right)$ as markers of malnutrition. The AUC was found to be $0.69(95 \%$ CI $0.39,1.00)$ for albumin $(P=0.12)$ and 0.74 (95\% CI $0 \cdot 67,0 \cdot 81)$ for BMI $(P<0 \cdot 0001)$, indicating that the MNA test is relatively accurate in detecting malnourished people according to BMI but not according to serum albumin.

The AUC, which represents the overall accuracy of the total MNA score as a test for detecting malnutrition and the risk of malnutrition, was $0 \cdot 8$ (95\% CI $0 \cdot 7,0 \cdot 8$; $P<0 \cdot 001)$.

The sensitivity and specificity of the MNA according to its established cut-off points were $82 \%$ and $63 \%$, respectively. Positive-predictive value was $35 \%$ and negative-predictive value was $93 \%$. By using the Youden index the best cut-off point to detect malnourished and at risk of malnutrition in the present study was 22 , with sensitivity of $88 \%$, specificity of $62 \%$, positive-predictive value of $57 \%$ and negative-predictive value of $89 \%$, respectively.

\section{Discussion}

A simple and clinically useful instrument for the nutritional screening of older patients is very important to prevent, detect and treat undernutrition, to prevent

Table 4 Correlations between total MNA score and nutritional status criteria (Spearman rank correlation coefficients, $r_{\mathrm{S}}$ ) among 221 elderly patients, Tehran, 2008

\begin{tabular}{lccr}
\hline Criterion & $n$ & $r_{\mathrm{S}}$ & $P$ value \\
\hline BMI & 216 & 0.46 & $<0 \cdot 001^{*}$ \\
Mid-arm circumference & 221 & 0.47 & $<0.001^{*}$ \\
Calf circumference & 221 & 0.55 & $<0.001^{*}$ \\
Serum albumin & 180 & 0.19 & $0.008^{*}$ \\
\hline
\end{tabular}

MNA, Mini Nutritional Assessment. ${ }^{\star} P<0.05$ is significant.

Table 3 Item-total score correlations (Spearman rank correlation coefficients, $r_{\mathrm{s}}$ ) for Mini Nutritional Assessment among 221 elderly patients, Tehran, 2008

\begin{tabular}{|c|c|c|c|}
\hline Item no. & Item content & $r_{\mathrm{S}}$ & $P$ value \\
\hline 1 & Changes in dietary intake & $0 \cdot 29$ & $<0.001^{*}$ \\
\hline 2 & Weight loss & $0 \cdot 18$ & $0.006^{*}$ \\
\hline 3 & Mobility & $0 \cdot 34$ & $<0.001^{*}$ \\
\hline 4 & Psychological stress or acute disease & $0 \cdot 12$ & 0.062 \\
\hline 5 & Neuropsychological problems & 0.29 & $<0.001^{*}$ \\
\hline 6 & BMI & $0 \cdot 20$ & $0.002^{*}$ \\
\hline 7 & Lives independently & -0.029 & 0.66 \\
\hline 8 & More than 3 prescription drugs per day & 0.076 & $0 \cdot 26$ \\
\hline 9 & Pressure sores or skin ulcers & 0.00 & 0.99 \\
\hline 10 & Full meals per day & 0.06 & 0.35 \\
\hline 11 & Protein intake & 0.004 & 0.95 \\
\hline 12 & Fruits or vegetables & $0 \cdot 11$ & 0.08 \\
\hline 13 & Fluid & $0 \cdot 19$ & $0.004^{*}$ \\
\hline 14 & Mode of feeding & $0 \cdot 12$ & 0.07 \\
\hline 15 & Self-view of nutritional status & 0.30 & $<0.001^{*}$ \\
\hline 16 & Health status in comparison with other people of the same age & 0.41 & $<0.001^{*}$ \\
\hline 17 & Mid-arm circumference & 0.36 & $<0.001^{*}$ \\
\hline 18 & Calf circumference & 0.41 & $<0.001^{*}$ \\
\hline
\end{tabular}

${ }^{*} P<0.05$ is significant. 
Table 5 Comparison of MNA scores between known groups (based on BMI, serum albumin and skin ulcers) among 221 elderly patients, Tehran, 2008

\begin{tabular}{|c|c|c|c|c|c|c|c|c|}
\hline \multirow[b]{2}{*}{ Groups with expected low scores } & \multirow[b]{2}{*}{$n$} & \multicolumn{2}{|c|}{ MNA score } & \multirow[b]{2}{*}{ Groups with expected high scores } & \multirow[b]{2}{*}{$n$} & \multicolumn{2}{|c|}{ MNA score } & \multirow[b]{2}{*}{$P$ value } \\
\hline & & Mean & SD & & & Mean & SD & \\
\hline $\mathrm{BMI}<24 \mathrm{~kg} / \mathrm{m}^{2}$ & 95 & $22 \cdot 2$ & $3 \cdot 5$ & $\mathrm{BMI} \geq 24 \mathrm{~kg} / \mathrm{m}^{2}$ & 121 & $24 \cdot 8$ & $2 \cdot 2$ & $<0.001^{*}$ \\
\hline Serum albumin <3.5 g/dl & 5 & $22 \cdot 5$ & $3 \cdot 4$ & Serum albumin $\geq 3.5 \mathrm{~g} / \mathrm{dl}$ & 175 & $24 \cdot 0$ & $2 \cdot 9$ & 0.27 \\
\hline Pressure sores/skin ulcers & 9 & $22 \cdot 2$ & $4 \cdot 6$ & No pressure sores/skin ulcers & 212 & $23 \cdot 7$ & $3 \cdot 1$ & 0.39 \\
\hline
\end{tabular}

MNA, Mini Nutritional Assessment.

${ }^{*} P<0.05$ is significant.

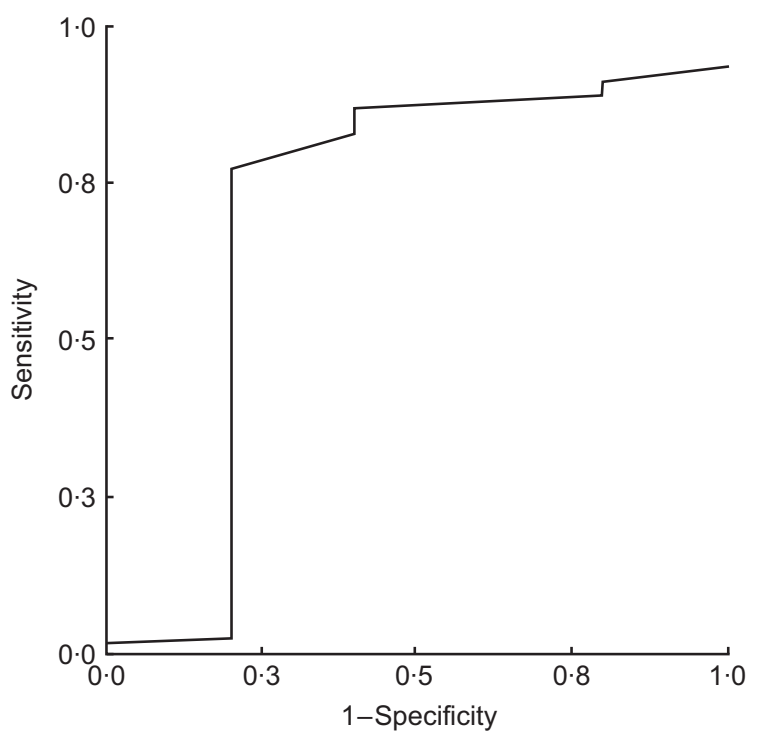

Fig. 1 Receiver-operating characteristic curve for the Mini Nutritional Assessment as a predictor of serum albumin level $<3.5 \mathrm{~g} / \mathrm{dl}$ among 221 elderly patients, Tehran, 2008. Diagonal segments are produced by ties

unwanted complications in the geriatric population. Thus the MNA was developed to be easy to use in identifying older patients who are nutritionally at risk. However, besides feasibility, reliability and validity are important factors for the screening outcome $e^{(11)}$.

The most extensively evaluated tool seems to be the MNA, which has been validated with older adult populations from the very frail to the healthy in a variety of settings, such as elective surgery ${ }^{(16)}$, outpatient clinics and nursing homes $^{(31)}$. Sensitivity and specificity have also been investigated in several studies ${ }^{(20,32)}$. In a review article by Guigoz, the MNA was mentioned as a screening and assessment tool with a reliable scale that clearly defines thresholds usable by health-care professionals ${ }^{(6)}$. The MNA is considered acceptable in terms of the time required for completion and its simplicity; although concerns have been raised about the transferability of the tool to countries in which it has not been validated, it is widely recognized as a useful nutritional assessment tool ${ }^{(33)}$.

In the present study, the MNA was used to detect malnutrition or risk of malnutrition in the elderly in Iran.

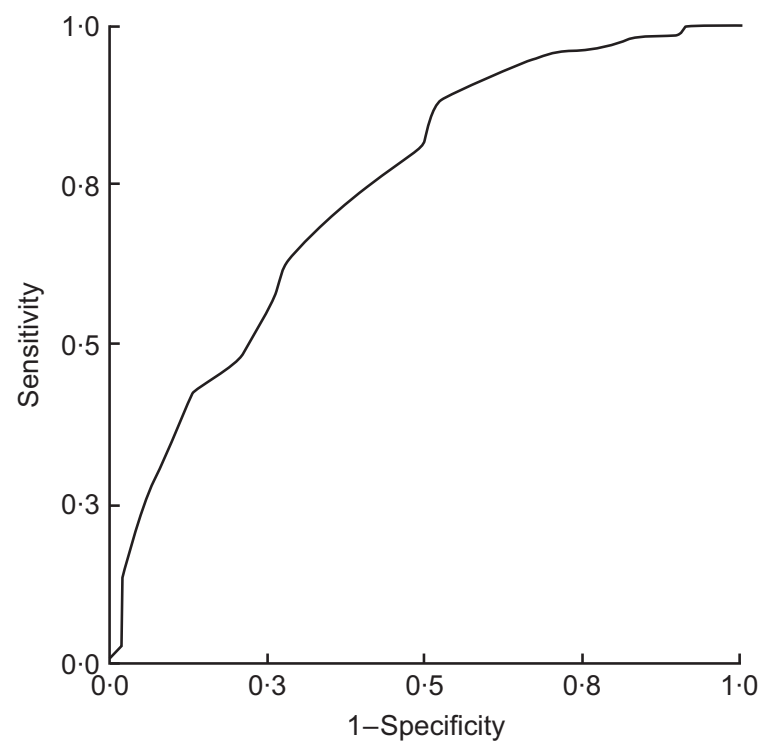

Fig. 2 Receiver-operating characteristic curve for the Mini Nutritional Assessment as a predictor of $\mathrm{BMI}<24 \mathrm{~kg} / \mathrm{m}^{2}$ among 221 elderly patients, Tehran, 2008. Diagonal segments are produced by ties

Anthropometric measurements including BMI, MAC and $\mathrm{CC}$, and serum albumin, were used as nutritional parameters. Although there are no generally accepted criteria for the diagnosis of malnutrition, these parameters have been widely used to evaluate nutritional status.

In the current study the MNA had a Cronbach's $\alpha$ value of $0 \cdot 6 ; \alpha$ should be between 0.70 and 0.90 to show a good reliability ${ }^{(20,31)}$.

We demonstrated that the total MNA score showed a good correlation with anthropometric markers and serum albumin; nevertheless, the AUC showed that the MNA can diagnose energy or energy-protein malnutrition but not protein malnutrition, of which low serum albumin is the marker. The significant difference between two BMI groups in mean MNA scores and the non-significant result between two groups according to serum albumin also confirm this result.

The MNA (using its established cut-off points) showed good sensitivity and specificity among the current study population, but the positive-predictive value of $35 \%$ caused a great false-positive prediction of malnutrition or 
at risk of malnutrition. This means that many cases with false-positive diagnoses of malnutrition might withdraw resources from those in real need of nutritional measures.

Using the best cut-off point for MNA scores, which was 22 (by the Youden index), to detect malnourished and at risk of malnutrition in the present study, resulted in a considerable increase in sensitivity and positivepredictive value (by $6 \%$ and $22 \%$, respectively). There were also slight decreases in specificity and negativepredictive value by $1 \%$ and $4 \%$, respectively.

The same result was seen in the study performed by Kuzuya et al. in Japan, which concluded that the full MNA cut-off point for malnutrition should be modulated for this population ${ }^{(34)}$.

To date, no ethnic-specific anthropometric targets exist; rather, these targets are derived from populations of US or European origin and are inappropriately applied to men and women of Asian descent. Ethnicity has been recognized as a significant modifier in anthropometric measurements ${ }^{(35)}$. In addition, the MNA contains dietary patterns that may differ across ethnicities ${ }^{(36)}$. Therefore, the MNA or cut-off point for malnutrition may not be a good fit for Asian populations, including Iranian elderly.

In elderly populations in Europe and the USA, a cut-off point below 24 for total MNA score, as an indicator of PEM or at risk of undernutrition, was found to have a sensitivity of $96 \%$, specificity of $98 \%$ and positive-predictive value of $97 \%{ }^{(5)}$. However, the same cut-off point yielded a much lower sensitivity and specificity among Iranian elders. Moreover, the latter study group consisted of the elderly from a charity foundation. Therefore, it is hard to consider this sample representative of the elderly Iranian population.

Further studies are required to evaluate the cut-off point for malnutrition and at risk of malnutrition in the elderly Iranian population. In addition, further investigation should be conducted to determine whether the MNA can correctly identify those elderly who are likely to benefit from nutritional support.

\section{Acknowledgements}

The study was supported by Tehran University of Medical Sciences and the Management Board of Kahrizak Charity Foundation. There is no conflict of interest in this study. B.A. contributed to writing the manuscript and analysing the data. F.S. contributed to analysing the data and writing the proposal. H.F. contributed to managing the research group and writing the proposal. M.M. and M.Gh. contributed to data gathering. B.L. made intellectual contributions to the study.

\section{References}

1. Sullivan DH, Sun S \& Walls RC (1999) Protein-energy undernutrition among elderly hospitalized patients: a prospective study. JAMA 281, 2013-2019.
2. Crogan NL \& Pasvogel A (2003) The influence of protein-calorie malnutrition on quality of life in nursing homes. J Gerontol A Biol Sci Med Sci 58, 159-164.

3. Aliabadi M, Kimiagar M, Ghayour-Mobarhan M et al. (2008) Prevalence of malnutrition in free living elderly people in Iran: a cross-sectional study. Asia Pac J Clin Nutr 17, 285-289.

4. Sadrivaan A, Sadrivaan B, Ghavamzadeh S et al. (2009) The nutritional status assessment of newly hospitalized patients at three units of governmental hospitals of Orumia, Iran (abstract). Presented at the 12th World Congress on Public Health, Istanbul, Turkey, 21 April-1 May 2009.

5. Guigoz Y, Vellas B \& Garry PJ (1996) Assessing the nutritional status of the elderly: the Mini Nutritional Assessment as part of the geriatric evaluation. Nutr Rev 54, S59-S65.

6. Guigoz Y (2006) The Mini Nutritional Assessment (MNA) review of the literature - what does it tell us? J Nutr Health Aging 10, 466-485.

7. Sullivan DH \& Walls RC (1998) Protein-energy undernutrition and the risk of mortality within six years of hospital discharge. J Am Coll Nutr 17, 571-578.

8. Persson MD, Brismar KE, Katzarski KS et al. (2002) Nutritional status using Mini Nutritional Assessment and subjective global assessment predict mortality in geriatric patients. J Am Geriatr Soc 50, 1996-2002.

9. Jeejeebhoy KN (2000) Nutritional assessment. Nutrition 16, $585-590$

10. Wright C (2002) Screening for undernutrition in the community. J Clin Nutr 4, 22-26.

11. Elia M, Zellipour L \& Stratton RJ (2005) To screen or not to screen for adult malnutrition? Clin Nutr 24, 867-884.

12. Joosten E, Vanderelst B \& Pelemans W (1999) The effect of different diagnostic criteria on the prevalence of malnutrition in a hospitalized geriatric population. Aging 11, 390-394.

13. Guigoz Y, Vellas B \& Garry PJ (1994) Mini nutritional assessment: a practical assessment tool for grading the nutritional state of elderly patients. Facts. Res Gerontol 4, $15-59$.

14. Gazzotti C, Albert A, Pepinster A et al. (2000) Clinical usefulness of the Mini Nutritional Assessment (MNA) scale in geriatric medicine. J Nutr Health Aging 4, 176-181.

15. Van Nes MC, Herrman FR, Gold G et al. (2001) Does the Mini Nutritional Assessment predict hospitalization outcomes in older people? Age Aging 30, 221-226.

16. Cohendy R, Gros T, Arnaud-Baltandier F et al. (1999) Preoperative nutritional evaluation of elderly patients: the Mini Nutritional Assessment as a practical tool. Clin Nutr 18, 345-348.

17. Vellas B, Hunt WC, Romero LJ et al. (1997) Changes in nutritional status and patterns of morbidity among freeliving elderly persons: a 10 year longitudinal study. Nutrition 13, 515-519.

18. Urteaga CR, Ramos RIH \& Atalah ES (2001) Validacion del criterio de evaluacion nutrioional global del adulto mayor. Rev Med Chil 129, 871-876.

19. Ek AC, Unosson M, Larsson J et al. (1996) Interrater variability and validity in subjective nutritional assessment of elderly patients. Scand J Caring Sci 10, 163-168.

20. Christensson L, Unosson M \& Ek A-C (2002) Evaluation of nutritional assessment techniques in elderly people newly admitted to municipal care. Eur J Clin Nutr 56, 810-818.

21. Charney P, Escott-Stump S \& Mahan LK (2000) Nutritional diagnosis and intervention. In Krause's Food, Nutrition and Diet Therapy, 11th ed., p. 433 [LK Mahan and S EscottStump, editors]. Philadelphia, PA: Elsevier.

22. Hamwi GJ (1964) Changing dietary concepts. In Diabetes Mellitus: Diagnosis and Treatment, vol. 1, pp. 73-78 [TS Danowski, editor]. New York: ADA. 
23. Streiner DL \& Norman GR (2003) Health Measurement Scales. A Practical Guide to Their Development and Use, 3rd ed. Oxford: Oxford University Press.

24. Polit DF \& Beck CT (2004) Nursing Research: Principles and Methods, 7th ed. Philadelphia, PA: Lippincott Williams \& Wilkins.

25. Beck AM \& Ovesen L (1998) At which body mass index and degree of weight loss should hospitalized elderly patients be considered at nutritional risk? Clin Nutr 17, 195-198.

26. Smith JL, Wickiser AA, Korth LL et al. (1984) Nutritional status of an institutionalized aged population. J Am Coll Nutr 3, 13-25.

27. Constans T, Bacq Y, Brechot JF et al. (1992) Protein-energy malnutrition in elderly medical patients. J Am Geriatr Soc 40, 263-268.

28. Fletcher RW \& Fletcher SW (2005) Clinical EpidemiologyThe Essentials, 4th ed. Philadelphia, PA: Lippincott Williams \& Wilkins.

29. Hanley JA \& McNeil BJ (1983) A method of comparing the areas under receiver operating characteristic curves derived from the same cases. Radiology $\mathbf{1 4 8}, 839$.
30. Youden WJ (1950) Index for rating diagnostic test. Cancer 3, 320-335.

31. Vellas B, Guigoz Y, Garry PJ et al. (1999) The Mini Nutritional Assessment (MNA) and its use in grading the nutritional state of elderly patients. Nutrition 15, 116-122.

32. Donini LM, De Felice MR, Tassi L et al. (2002) 'Proportional and objective score' for the Mini Nutritional Assessment in long-term geriatric care. J Nutr Health Aging 6, 141-146.

33. Chumlea WMC (1999) The state of the Mini Nutritional Assessment? Nutrition 15, 159-161.

34. Kuzuya M, Kanda S, Koike T et al. (2005) Evaluation of Mini-Nutritional Assessment for Japanese frail elderly. Nutrition 21, 498-503.

35. Wang J, Thornton JC, Russell M et al. (1994) Asians have lower body mass index (BMI) but higher percent body fat than do whites: comparisons of anthropometric measurements. Am J Clin Nutr 60, 23-28.

36. Maskarinec G, Novotny R \& Tasaki K (2000) Dietary patterns are associated with body mass index in multiethnic women. J Nutr 130, 3068-3072. 